A】ll06 979134

PUBIICATIONS

NBSIR 83-1689

\title{
APPROXIMATE FORMULAS FOR THE FAR FIELDS AND GAIN OF OPEN-ENDED RECTANGULAR WAVEGUIDE
}

Arthur D. Yaghjian

National Bureau of Standards

U.S. Department of Commerce

Boulder, Colorado 80303

May 1983 

NBSIR 83-1689

$\rightarrow$

\section{APPROXIMATE FORMULAS}

FOR THE FAR FIELDS AND GAIN OF

OPEN-ENDED RECTANGULAR

WAVEGUIDE

Arthur D. Yaghjian

Electromagnetic Fields Division

National Engineering Laboratory

National Bureau of Standards

U.S. Department of Commerce

Boulder, Colorado 80303

May 1983

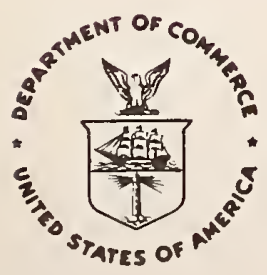

U.S. DEPARTMENT OF COMMERCE, Malcolm Baldrige, Secretary 



\section{CONTENTS}

Page

I. INTRODUCTION................................ 1

I I. DERIVATION OF FAR-FIELD FORMULAS...................... 3

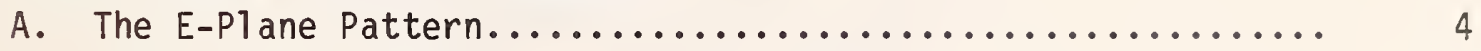

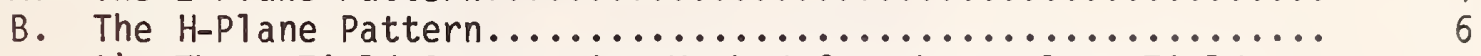

1) The E-Field Integration Method for the H-Plane Fields... 6

2) The Fringe Current Method for the H-Plane Fields....... 8

III. DETERMINATION OF ON-AXIS GAIN.................... 14

IV. CONCLUSION................................. 15

APPENDIX I ...................................... 17

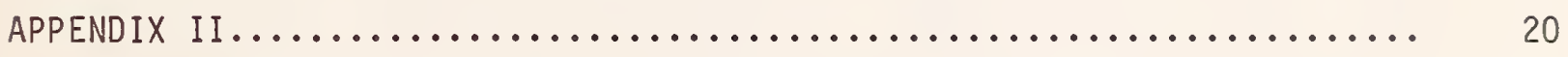

ACKNOWLEDGMENT.................................... 23

REFERENCES........................................ 24 



\title{
Approximate Formulas for the Far Fields and Gain of Open-Ended Rectangular Waveguide
}

\author{
Arthur D. Yaghjian \\ Electromagnetic Fields Division \\ National Bureau of Standards \\ Boulder, Colorado 80303
}

Approximate formulas are derived for the far field and gain of standard, open-ended, unflanged, rectangular waveguide probes operating within their recommended usable bandwidth of frequencies. (Such probes are commonly used in making probe-corrected near-field antenna measurements.) The formulas, which yield forward far-field power patterns and on-axis gains of $X$-band and 1 arger waveguide probes to within about $2 \mathrm{~dB}$ and $0.2 \mathrm{~dB}$ accuracy, respectively, assume ( $\sin \phi-\cos \phi)$ azimuthal angular dependence and an E-plane pattern given by the traditional aperture integration of the $T E_{10}$ mode $E$ - and $H$-fields in the Stratton-Chu equations. The H-plane pattern is estimated by two different methods. The first, and less accurate, method uses a purely Efield aperture integration to estimate the H-plane pattern. The second, more accurate, method uses an electric field integral equation (EFIE) to show that fringe currents near the shorter edges of the guide can be well-approximated by isotropic line sources. The amplitude of these line sources is then determined more simply by equating the total power radiated into free space to the net input power to the waveguide.

Key words: edge diffraction, fringe current, physical optics, radiation pattern, rectangular waveguide.

\section{INTRODUCTION}

Most near-field antenna measurements are made using open-ended, unflanged, rectangular waveguide probes. They are simple, rugged, and inexpensive to produce (or reproduce), they have a broad far-field power pattern that 
contains no nulls in the forward hemisphere over their recommended usable bandwidth, they scatter relatively little radiation back to the test antenna being measured, and they have a respectable gain of about $6-8 \mathrm{~dB}$. However, unlike far-field measurements where only the on-axis gain of the probe is required, near-field measurements require, in general, the complex far field in the forward hemisphere of the probe in order to compensate for its presence in the near field. Specifically, for each microwave frequency at which the test antenna is to be measured, the appropriate standard rectangular waveguide probe is chosen and its far-field pattern, gain, and reflection coefficient are measured as a necessary part of the complete near-field measurement procedure.

The reflection coefficient can be measured accurately and rapidly over the waveguide bandwidth using, e.g., an automatic network analyzer, but the measurement of probe pattern and gain is a rather tedious, time-consuming operation, especially when the fields of the test antenna are desired at several frequencies for which the probe has not been previously calibrated.

To alleviate this chore of measuring the probe far fields, at least when extremely high accuracy is not required, we have derived reasonably accurate formulas for the far-field pattern and gain of open-ended, standard rectangular waveguides over their recommended usable bandwidths. For example, comparison with measured data indicates that equations (2) and (8) inserted into (1) approximate the forward far-field power pattern and on-axis gain of $X$-band (WR-90) and larger standard waveguide to within about $2 \mathrm{~dB}$ and $0.2 \mathrm{~dB}$ accuracy, respectively (see figures 3, 6, and 7), the maximum pattern error occurring near $90^{\circ}$ off boresight. This accuracy represents a significant improvement over previous approximate formulas derived either entirely by aperture integration with the Stratton-Chu formulas [1, Sec. 8.14; 2], or by a first order geometrical edge diffraction technique [3]. Both of the previous methods yield far-field power patterns several dB too high near much of the $\mathrm{H}$ plane cut, and predict on-axis gain values which are typically 2 to 3 dB too low. For Ku-band (WR-62) and smaller standard waveguides, the approximate formulas derived in this paper may become less accurate because the wall thickness of these smaller waveguides becomes an appreciable fraction of their height. 


\section{DERIVATION OF FAR-FIELD FORMULAS}

We take a semi-empirical approach to approximate the far fields and absolute gain of open-ended waveguide. In principle, one could obtain a numerical solution for the fields everywhere by solving an electric field integral equation (EFIE) for the currents over the surface of the semi-infinite waveguide. And, indeed, we use this EFIE approach (formulated in Appendix I) in Sec. II-B-2 to estimate the fringe current contribution to the $\mathrm{H}-\mathrm{pl}$ ane fields. However, we do not want to finally rely on numerical integral equation solutions because for 3-D problems like the open-ended rectangular waveguide, they demand an exorbitant amount of computer time and storage to achieve the desired accuracy, they are not nearly as simple to use as the approximate formulas, and they inspire less physical insight.

The geometry of the open-ended rectangular waveguide is shown in figure 1. The inner dimensions of the waveguide are given by width $a$ and height $b$. The perfectly conducting waveguide walls are assumed to have a thickness small enough compared to the smaller dimension $b$ so that the finite thickness of the walls can be ignored. The origin 0 of the $x y z$ rectangular coordinate system is chosen at the center of the open end (aperture or mouth) of the waveguide, and the spherical coordinates of the position vector $\bar{r}$ to any point in space is denoted by $(r, \theta, \phi)$. The waveguide operates at a frequency, $f$, that lies within the recommended usable bandwidth of the $T E_{10}$ mode (electric field in the $y$ direction). For example, for standard $X$-band waveguide, the recommended usable bandwith ranges from 8.2 to $12.4 \mathrm{GHz}$, or equivalently the free-space wavelength, $\lambda$, ranges from $1.6 a$ to $1.06 a, a=2.286 \mathrm{~cm}$.

Consider the far field of the waveguide expressed as a sum of spherical multipoles located at the origin 0 . Because the transverse dimensions of the waveguide are less than a wavelength, only the multipoles of lower order azimuthal $(\phi)$ dependence will contribute significantly to the far field. Moreover, because all but the first-order $(\sin \phi-\cos \phi)$ dependent multipoles have a null in the on-axis $(z)$ direction, one would expect a significant contribution only from these first-order multipoles. Under this assumption and the symmetry of the rectangular waveguide excited by the $T E_{10}$ mode, the far 
fields of the open-ended waveguide can be expressed approximately in the following simple form [1, Sec. 7.11$]$ :

$$
\begin{aligned}
& \bar{E}(\bar{r} \rightarrow \infty)=\frac{e^{i k r}}{k r}\left[E_{E}(\theta) \sin \phi \hat{e}{ }_{\theta}+E_{H}(\theta) \cos \phi \hat{e}{ }_{\phi}\right] \\
& \bar{H}(\bar{r} \rightarrow \infty)=\frac{e^{i k r}}{k r} \frac{1}{Z_{0}}\left[E_{E}(\theta) \sin \phi \hat{e}_{\phi}-E_{H}(\theta) \cos \phi \hat{e}_{\theta}\right],
\end{aligned}
$$

where $e^{-i \omega t}$ time dependence has been suppressed $(\omega=2 \pi f, k=2 \pi / \lambda)$, and $z_{0}$ is the impedance of free space. Equations (1) immediately reduce the problem of finding the far fields to that of finding the E-plane and H-plane patterns, $E_{E}(\theta)$ and $E_{H}(\theta)$, respectively. Indication that equations (1) represent the far fields $\stackrel{H}{H}$ of the open-ended waveguide to a rather good approximation in the forward hemisphere can be seen from figure 2 which plots the measured power patterns of an X-band waveguide at $9.32 \mathrm{GHz}$ for $\phi=0,45$, and 90 degrees, and compares the 45-degree pattern with $\frac{1}{2}\left[\left|E_{E}\right|^{2}+\left|E_{H}\right|^{2}\right]$, i.e., with the power pattern predicted by (1) from the measured $\mathrm{E}$ - and $\mathrm{H}$-plane patterns. In particular, the $\phi$-dependence of (1) conforms more closely to the measured power patterns than the "separable dependence" obtained from aperture integration with the Stratton-Chu formulas [2] or through first-order geometrical theory of edge diffraction [3]. The approximation (1) would not, however, be expected to give very accurate polarization ratios for wide $\theta$ angles near $\phi=$ $45^{\circ}$.

\section{A. The E-Plane Pattern}

The E-plane pattern, $E(\theta)$, is predicted quite accurately by inserting the $E-$ and $H$-fields of the propagating $T E_{10}$ mode into the Stratton-Chu formulas [1,Sec. 8.14] and integrating over the mouth of the open-ended waveguide [2]. Risser explained in [2], and we prove in Appendix I, that this StrattonChu integration of the $\mathrm{TE}_{10}$ mode is equivalent to integrating over the physical optics current, i.e., the truncated interior surface currents $\bar{k}_{10}$ of the propagating $\mathrm{TE}_{10}$ mode (incident plus reflected). In other words, the resulting far fields obtained from the Stratton-Chu formulas neglect only the 
evanescent mode currents inside the waveguide and the surface currents on the outside of the waveguide. Because the sum of both these currents equal the total surface current less the physical optics current, we can simply say that the Stratton-Chu formulas applied to the $\mathrm{TE}_{10}$ mode neglect only the Ufimtsev "nonuniform" or "fringe" currents [12].

Although these fringe currents can contribute appreciably to the absolute value of the field, they have a minor secondary effect in the broad E-plane pattern which remains well above $-10 \mathrm{~dB}$ for all angles $\theta$. Thus, for $E_{E}(\theta)$ we simply use the classical result of the aperture integration with the StrattonChu formulas [2]

$$
E_{E}(\theta)=A_{E} \frac{\left[1+\frac{\beta}{k} \cos \theta+\Gamma\left(1-\frac{\beta}{k} \cos \theta\right)\right]}{\left[1+\frac{\beta}{k}+\Gamma\left(1-\frac{\beta}{k}\right)\right]} \frac{\sin \left(\frac{k b}{2} \sin \theta\right)}{\frac{k b}{2} \sin \theta} .
$$

The normalized propagation constant $\beta / \mathrm{k}$ for the $T E_{10}$ mode equals $\sqrt{1-\left(\frac{\pi}{\mathrm{ka}}\right)^{2}}$ and the reflection coefficient of the $T E_{10}$ mode from the end of the waveguide is denoted by $\Gamma$. The constant $A_{E}$ which remains arbitrary at this point in the derivation is eventualiy related to the amplitude of the incident $T E_{10}$ mode through equation (9). The factor $\left[1+\frac{\beta}{k}+\Gamma\left(1-\frac{\beta}{k}\right)\right]$ in the denominator of (2) is included merely to simplify the normalization at $\theta=0$.

Since (2) could be obtained by integrating the truncated $\mathrm{TE}_{10}$ surface currents, it remains a valid approximation for $\theta$ in the back as well as the forward hemisphere. However, (2) becomes a better approximation in the forward hemisphere when $\Gamma$ is set equal to zero because zeroing $\Gamma$ tends to compensate in the forward-hemisphere E-plane for the neglected fringe currents. Thus, whenever (2) is used in the region of most interest, i.e., the forward hemisphere, $\Gamma$ will be assumed zero. If (2) is used in the back hemisphere, a more accurate pattern results by including the reflection coefficient beyond $\theta$ equal to about $105^{\circ}$.

In figure 3 the amplitude and phase of $E_{E}(\theta)$ in the forward hemisphere is plotted from (2) and compared with NBS (National Bureau of Standards) measured data for X-band waveguide at $9.32 \mathrm{GHz}$ and $\mathrm{L}$-band (WR-650) waveguide at 1.0 
$\mathrm{GHz}$. The close agreement between the theoretical and experimental curves was somewhat unexpected for the L-band guide because the $1 \mathrm{GHz}$ frequeny is approaching the $0.908 \mathrm{GHz}$ cut-off frequency and lies well below the lowest recommended usable frequency of $1.12 \mathrm{GHz}$.

\section{B. The H-Plane Pattern}

The H-plane pattern, $E_{H}(\theta)$, is much narrower than the E-plane pattern and, consequently, it is more strongly influenced by the fringe currents neglected in the Stratton-Chu aperture integration. Specifically, the Stratton-Chu integration with the $\mathrm{TE}_{10}$ mode yields an $\mathrm{H}-\mathrm{plane}$ pattern much broader than the measured pattern. And this is true whether the reflection coefficient $\Gamma$ of the $T E_{10}$ mode is included or not. Thus we are left with finding a method that will predict accurately and simply the H-plane pattern of open-end waveguide over the recommended usable bandwidth.

Two separate methods will be developed for determining the H-plane pattern. The first method, referred to as the E-field integration method, does not require the reflection coefficient $\Gamma$, but produces a valid $H$-plane pattern over the forward hemisphere only, and erroneously becomes exactly zero at $\theta=$ $90^{\circ}$. The second, a more complicated method referred to herein as the fringe current method, does require the reflection coefficient and yields an accurate $H$-plane pattern for all $\theta$--front and back hemisphere. This second method also yields slightly more reliable values of on-axis gain.

\section{1) The E-Field Integration Method for the H-Plane Fields}

As explained above, aperture integration of the Stratton-Chu formulas with the electric and magnetic fields of the $T E_{10}$ mode neglects the fringe currents and thereby produces much too broad an H-plane pattern. However, the Stratton-Chu formulas are not the only expressions that can be used to predict the far fields from an aperture integration of the fields of the $\mathrm{TE}_{10}$ mode. In particular, the electric or magnetic far field in the forward hemisphere can be expressed respectively as a double Fourier transform of the electric or magnetic field alone over the infinite plane just in front of the mouth of the 
waveguide. Concentrating on the electric field, for reasons that will become clear in the next paragraph, we express the H-plane far field as [4]

$$
\overline{\substack{\mathrm{E}(r \rightarrow \infty, \theta, \phi=0) \\ 0 \leqslant \theta \leqslant \pi / 2}}=\frac{e^{i k r}}{k r} \frac{k^{2}}{2 \pi} \hat{e}_{r} \times \hat{e}_{z} \times \int_{-\infty}^{\infty} \int_{-\infty}^{\infty} \bar{E}\left(\bar{R}^{\prime}\right) e d x^{\prime} d y^{\prime},
$$

where $\bar{R}^{\prime}=x^{\prime} \hat{e}_{x}+y^{\prime} \hat{e}_{y}$ is the integration vector from the origin 0 to an area element $d x^{\prime} d y^{\prime}$ in the plane of integration.

Now the electric and magnetic field $\left(\bar{E}_{10}\right.$ and $\left.\bar{H}_{10}\right)$ of the $T E_{10}$ mode is given by

$$
\begin{aligned}
& \bar{E}_{10}=E_{0}(1+\Gamma) \cos \left(\frac{\pi x}{a}\right) \hat{e}_{y} \\
& \bar{H}_{10}=-\frac{E_{0}}{Z_{0}}\left[(1-\Gamma) \frac{\beta}{k} \cos \left(\frac{\pi x}{a}\right) \hat{e}_{x}+\frac{\pi}{i k a}(1+\Gamma) \sin \left(\frac{\pi x}{a}\right) \hat{e}_{z}\right],
\end{aligned}
$$

where $E_{0}$ is the arbitrary amplitude of the incident $T E_{10}$ mode. Note that the E-field has but one component that goes to zero at the edges $x= \pm a / 2$. This suggests there will be little difference between the average $x$-variation of the electric field just in front of the mouth of the waveguide and that of the $\mathrm{TE}_{10}$ mode. In other words, for the purpose of determining a reasonably accurate H-plane pattern, the E-field on the integration plane in (3) can be approximated by the $\bar{E}_{10}$ of $(4 a)$ over the mouth or aperture of the waveguide and zero outside. Performing this simple integration of (3) gives an approximate $\mathrm{H}$-plane pattern valid in the forward hemisphere

$$
\underset{\substack{H \\ 0 \leqslant \theta \leqslant \frac{\pi}{2}}}{E_{E}(\theta)}=A_{E}\left(\frac{\pi}{2}\right)^{2} \cos \theta \frac{\cos \left(\frac{k a}{2} \sin \theta\right)}{\left[\left(\frac{\pi}{2}\right)^{2}-\left(\frac{k a}{2} \sin \theta\right)^{2}\right]} .
$$

The constant $A_{E}$ in $(5)$ is the same as in $(2)$ because $E_{E}(\theta)$ must equal $E_{H}(\theta)$ along the common axis $\theta=0$. The same integration with $E_{10}$ does not and would not be expected to yield an accurate E-plane pattern because the E-plane 
pattern is determined by the y-variation of the aperture field which differs significantly from that of the incident $\bar{E}_{10}$ mode field through diffraction from the edges of the waveguide at $y= \pm b / 2$. Also, substituting $\bar{H}_{10}$ from (4b) into the formula for the magnetic field corresponding to (3) yields a very poor approximation for both the E- and H-plane far fields. This, of course, is no surprise because the $\bar{H}_{10}$ field does not become zero at any of the waveguide edges.

Figure 4 compares the amplitude and phase curves for the H-plane fields in the forward hemisphere calculated from (5), measured at NBS, and calculated from the following Stratton-Chu expression for the $\mathrm{H}$-plane pattern [2]

$$
E_{H}(\theta)=A_{H} \frac{\left[\left(\cos \theta+\frac{\beta}{k}\right)+\Gamma\left(\cos \theta-\frac{\beta}{k}\right)\right]}{\left[\left(\frac{\pi}{2}\right)^{2}-\left(\frac{k a}{2} \sin \theta\right)^{2}\right]} \cos \left(\frac{k a}{2} \sin \theta\right),
$$

where $A_{H}=-i k^{2}$ ab $E_{0} / 8$.

The H-plane fields predicted by (5) agree well with the measured data even near the L-band cut-off frequency, and they are much closer (on a linear scale) to the measured values than those predicted by the result (6) of the Stratton-Chu formulas.

2) The Fringe Current Method for the H-Plane Fields

Although the integrated E-field expression (5) for the H-plane pattern is much more accurate in the forward hemisphere than the previous expressions $[2,3]$, it has a couple of shortcomings that the E-plane expression (2) does not have. First, the expression (5) does not remain valid (or accurate) in the back hemisphere because it was derived from (3) which applies only to the front, free-space hemisphere. Thus, when the constant $A_{E}$ is related to total power radiated, the $\mathrm{H}$-plane pattern in the back hemisphere must be estimated and this estimation introduces an additional, albeit small, inaccuracy into the determination of gain (see Sec. III). Secondly, the expression (5) 
approaches zero like $\cos \theta$ as $\theta$ approaches $90^{\circ}$, and predicts too 1 ow an $\mathrm{H}$-plane pattern for $\theta$ between about $70^{\circ}$ and $90^{\circ}$. Fortunately for all near-field antenna measurements, except those made with the probe extremely close to the test antenna, the pattern of the probe beyond $70^{\circ}$ does not intercept the test antenna and thus does not affect the probe correction $[5,6,7]$. However, for the sake of those rare exceptions as well as for slightly more reliable estimates of gain, we derive an alternative expression to (5) for the $H$-plane pattern that remains a good approximation for all values of $\theta$.

To derive this alternative expression, we return to a formulation of the open-ended waveguide problem in terms of surface currents. As mentioned above, discussed by Risser in [2], and proven in Appendix I, the far fields of the open-ended waveguide obtained by an aperture integration of the $T E_{10}$ mode fields in the Stratton-Chu formulas neglect only the fringe currents (total current minus physical optics current, or equivalently, evanescent mode currents plus the exterior surface currents). From the symmetry of the rectangular waveguide and $T E_{10}$ mode, only y-directed currents contribute to the $\mathrm{H}$ plane fields. Thus, equation (6) neglects only the fringe currents along the $x= \pm a / 2$ edges of the open end of the waveguide (provided the finite waveguide wall thickness of the waveguide can be ignored). By estimating the contribution to the $\mathrm{H}-\mathrm{pl}$ ane of these fringe currents and adding it to the Stratton-Chu result (6), an alternative formula to (5) for the $H$-plane pattern is obtained that remains a reasonably accurate approximation for all values of $\theta$.

We first tried to obtain a good estimate of the $y$-directed fringe currents by looking at the $x=+a / 2$ or $-a / 2$ edge as part of the infinite edge of a half plane. The exciting field was taken as the incident and reflected plane wave produced by the incident and reflected $T E_{10}$ mode. Since (6) is an exact closed-form expression for the H-plane contribution from the physical optics current ( $i . e .$, the truncated $T E_{10}$ mode current), the only additional information we needed was the far fields of the fringe currents of the half plane E-wave problem. For this problem, these fringe currents produce far fields that are also expressible by exact, nonsingular, closed form expressions derived by Ufimtsev for the general wedge [12] and recast for the half plane into simplified form in Appendix II. This latter result is worth emphasizing--the far-field diffraction pattern of the Ufimtsev fringe currents for 
the E-wave incident upon the half plane is given exactly with no singularities* by the simple function (AB) involving only sines and cosines of the observation angle and the angle of plane-wave incidence.

Appendix II shows that this half-plane fringe current correction works very well for the open-ended parallel-plate waveguide excited by the $T E_{10}$ mode, but unfortunately for the open-ended rectangular waveguide, it did not bring the predicted $\mathrm{H}-\mathrm{plane}$ pattern to within the desired 1 or $2 \mathrm{~dB}$ accuracy of the measured pattern at the wider angles of observations. The reason for the greater inaccuracy in the case of the rectangular waveguide is simply that the top and bottom edges of the rectangular waveguide strongly influence the edge diffraction from the $x= \pm a / 2$ side edges, and produce fringe currents significantly different from those of the half-plane problem. One may be able to get a better estimate of the fringe current contribution by examining the mutual interactions between the adjacent edges of the rectangular waveguide; but such an analysis amounts to determining the diffraction coefficients for the open corner problem, which eludes a closed-form solution.

We were finally able to obtain an accurate estimate of the fringe currents on the $x= \pm a / 2$ sides of the rectangular waveguide from a numerical solution to the EFIE applied to the open-ended rectangular waveguide. A1though the detailed formulation of the EFIE solution is given in Appendix I, just one major result of that solution is used here to modify the $\mathrm{H}$-plane pattern (6). Namely, the numerical EFIE solution revealed that the fringe currents at $x= \pm a / 2$ contributed to the $H$-plane pattern like line sources that radiate isotropically with $\theta$. That is, the contribution to the $H$-plane pattern from the fringe current can be approximated simply by that of equal isotropic line sources at $(x= \pm a / 2, z=0)$, or specifically by

$$
A_{H} C_{0} \cos \left(\frac{k a}{2} \sin \theta\right) \text {, }
$$

\footnotetext{
*In making this statement, we are assuming that the angles of incidence and scattering do not both equal the grazing (edge on) angle, $\theta=\alpha= \pm \pi$ in (A8). For the plane waves composing the $\mathrm{TE}_{10}$ mode in the waveguide, $\alpha$ is never too close to $\pm \pi$, when operating within recommended bandwidth.
} 
where $C_{0}$ is a positive real constant. Adding (7) to (6) then determines the $\mathrm{H}-\mathrm{pl}$ ane pattern derived by the fringe current method

$$
E_{H}(\theta)=A_{H}\left[\frac{\left(\cos \theta+\frac{\beta}{k}\right)+\Gamma\left(\cos \theta-\frac{\beta}{k}\right)}{\left(\frac{\pi}{2}\right)^{2}-\left(\frac{k a}{2} \sin \theta\right)^{2}}+c_{0}\right] \cos \left(\frac{k a}{2} \sin \theta\right) .
$$

The constant $A_{H}$ is defined under (6) and the $H$-plane constant $A_{E}$ in is related to $A_{H}$ by $E_{E}(0)=E_{H}(0)$, or

$$
A_{E}=A_{H}\left\{\left(\frac{2}{\pi}\right)^{2}\left[\left(1+\frac{\beta}{k}\right)+\Gamma\left(1-\frac{\beta}{k}\right)\right]+C_{0}\right\} .
$$

The positive real constant $C_{0}$, which depends on the frequency and the waveguide dimensions, remains the one unknown in the fringe current expressions (8) and (9). It can be determined in a number of ways, including directly from the EFIE program discussed in Appendix II. A simpler way to find $C_{0}$, however, is to equate the radiated power $P_{r}$ determined from the far-field (1) (with (2) and (8) inserted) to the total input power $P_{0}$ determined from the $\mathrm{TE}_{10}$ mode fields (4), and solve the resulting quadradic equation for $C_{0}$. From (1) and (4), $P_{0}$ and $P_{r}$ are found to be, respectively,

$$
\begin{aligned}
& P_{0}=\frac{a b\left|E_{0}\right|^{2}\left(1-|\Gamma|^{2}\right) \beta / k}{4 Z_{0}} \\
& P_{r}=\frac{\pi}{2 Z_{0} k^{2}} \int_{0}^{\pi}\left(\left|E_{E}(\theta)\right|^{2}+\left|E_{H}(\theta)\right|^{2}\right) \sin \theta d \theta
\end{aligned}
$$

Because the resistive loss in the waveguide walls is negligible, $P_{r}$ must equal $P_{0}$. Upon equating $(10 a)$ and $(10 b)$, we find 


$$
a_{q} c_{0}^{2}+2 b{ }_{q} c_{0}+c_{q}=0
$$

or

$$
c_{0}=\frac{-b q+\sqrt{(b q)^{2}-c_{q} a_{q}}}{a_{q}}
$$

where $a_{q}, b_{q}$, and $c_{q}$ are constants determined by integrals of the $\mathrm{E}$ - and $\mathrm{H}-$ plane patterns, (2) and (8). These integrals were evaluated numerically and $C_{0}$ from (11b) was determined for X-, C-, and L-band waveguides (WR-90, WR-187, WR-650). The on-axis gain $G_{0}$ is obtained directly from $C_{0}$ through the definition

$$
G_{0} \equiv \frac{4 \pi r^{2}|\bar{E}(r \rightarrow \infty, \theta=0)|^{2}}{2 Z_{0} P_{0}}=\frac{8 \pi r^{2}|\bar{E}|^{2}}{\left|E_{0}\right|^{2} a b \frac{\beta}{k}\left(1-|\Gamma|^{2}\right)},
$$

from which we find, after inserting the on-axis far field from (2) or (8) into (12a)

$$
G_{0}=\frac{\pi k^{2} a b}{8 \frac{\beta}{k}(1-|\Gamma|)^{2}}\left|\left[1+\frac{\beta}{k}+\Gamma\left(1-\frac{\beta}{k}\right)\right]\left(\frac{2}{\pi}\right)^{2}+c_{0}\right|^{2} .
$$

Likewise, $C_{0}$ can be written in terms of the on-axis gain by solving (12b) for $C_{0}$ in terms of $G_{0}$,

$$
C_{0}=-R_{e}\left[C_{E}\right]+\sqrt{\left(R_{e}\left[C_{E}\right]\right)^{2}-\left(\left|C_{E}\right|^{2}-\frac{G_{0}}{A}\right)},
$$

where

$$
C_{E} \equiv\left[1+\frac{\beta}{k}+\Gamma\left(1-\frac{\beta}{k}\right)\right]\left(\frac{2}{\pi}\right)^{2}
$$




$$
A \equiv \frac{\pi k^{2} a b}{8 \frac{\beta}{k}\left(1-|\Gamma|^{2}\right)} .
$$

For $|\Gamma|(1-\beta / k) \ll(1+\beta / k),(12 c)$ reduces to

$$
C_{0} \approx-\left(1+\frac{\beta}{k}\right)\left(\frac{2}{\pi}\right)^{2}+\sqrt{\frac{G_{0}}{A}} .
$$

Since $(12 c)$ or (12d) are simple expressions for the constant $C_{0}$ as a function of gain, and since the predicted gain $G_{0}$ from $(12 b)$ is plotted in figure 7 for $X_{-}, C_{-}$, and L-band waveguides, $C_{0}$ will not be plotted explicitly. The complex reflection coefficients over the recommended usable bandwidth of the $X_{-}, C_{-}$, and $\mathrm{Ku}$-band waveguides, needed to determine $C_{0}$ from (11), were obtained from measurements with an automatic network analyzer at NBS. The amplitude and phase of these measured $\Gamma$ are shown in figure 5. (The L-band reflection coefficient was estimated by scaling the given Ku-band data, since b/a for Land $\mathrm{Ku}$-band waveguide are the same and L-band data was not immediately available. Of course, some error is introduced by this scaling because the wall thickness of $\mathrm{Ku}$ - and L-band waveguide do not scale like the inner dimensions.)

Using these measured reflection coefficients and the $C_{0}$ evaluated from (11), the amplitude and phase of the H-plane pattern (8) is plotted in figure 6 for the forward hemisphere of X-band waveguide at $9.32 \mathrm{GHz}$ and L-band waveguide at $1.0 \mathrm{GHz}$. These predicted $\mathrm{H}$-plane patterns (8) derived from the fringe current method agree well with measured patterns even near $\theta=90^{\circ}$ and, though not shown in figure 6 , with measured patterns over the back hemisphere as well. The disadvantages of using (8) instead of the H-plane expression (5) derived from the E-field method are the extra computations needed to evaluate $C_{0}$ and the need to supply the reflection coefficient $\Gamma$.

To determine $C_{0}$ from (11), the far-field integrals in (10b) must be evaluated over the full hemisphere. This was done numerically by simply converting the integrals to summations and adding on a computer. The reflec- 
tion coefficient in the E-plane (2) was assumed zero for $\theta \leqslant 105^{\circ}$ and taken as its measured value for $\theta>105^{\circ}$ (see Sec. II-A).

\section{DETERMINATION OF ON-AXIS GAIN}

Two approximations have been derived for the far fields of open-ended rectangular waveguides. The first uses equation (2) and (5), and the second uses equations (2) and (8) for the E- and H-plane patterns in (1). This section evaluates the absolute on-axis gain predicted by each approximation.

Because the resistive loss in the walls of the waveguide is negligible, the total radiated power $P_{r}$ must equal the net input power $P_{0}$, and the on-axis gain (defined in (12a)) becomes equal to the on-axis directivity:

$$
G_{0}=\frac{4 \pi r^{2}|\bar{E}(r \rightarrow \infty, \theta=0)|^{2}}{2 Z_{0} P_{r}},
$$

where $P_{r}$ is expressed by (10b) in terms of the E- and H-plane patterns. Using (2) with $\Gamma=0$ for the E-plane pattern and (5) for the H-plane pattern, (13) can be expressed explicitly as

$G_{0}=4 / \int_{0}^{\pi}\left\{\left[\frac{\left(1+\frac{\beta}{k} \cos \theta\right) \sin \left(\frac{k b}{2} \sin \theta\right)}{\left(1+\frac{\beta}{k}\right) \frac{k b}{2} \sin \theta}\right]^{2}+\left[\left(\frac{\pi}{2}\right)^{2} \frac{\cos \theta \cos \left(\frac{k a}{2} \sin \theta\right)}{\left(\frac{\pi}{2}\right)^{2}-\left(\frac{k a}{2} \sin \theta\right)^{2}}\right]^{2}\right\} \sin \theta d \theta$. Note that the arbitrary constant A for the approximate formulas (2) and (5) cancels in the determination of gain from (14). If desired, the magnitude of $A_{E}$ for (2) and (5) can be written in terms of the coefficient $E_{0}$ of the incident $T E_{10}$ mode by simply equating $P_{0}$ and $P_{r}$ to get

$$
\left|A_{E}\right|^{2}=\frac{k^{2} a b\left(1-|\Gamma|^{2}\right) \frac{\beta}{k}}{2 \pi(\text { Denom })}\left|E_{0}\right|^{2}
$$

where "Denom" denotes the denominator of the right side of (14). 
It should be pointed out that the integral denominator in (14) was not evaluated exactly as it is written to get the gain values labeled by eq (14) in figure 7. As explained in Sec. II-B-1, the H-plane pattern (5) does not remain valid for the back hemisphere, and, in fact, becomes a very poor approximation beyond a $\theta$ of about $105^{\circ}$. Moreover, as explained in Sec. II-A, the E-plane pattern in the back hemisphere, unlike the forward hemisphere, remains accurate beyond about $105^{\circ}$ only if the reflection coefficient is included. Since we do not want to have to require the reflection coefficient when using the approximate formulas (2) and (5), both the E- and H-plane patterns in the integral denominator of (14) beyond $\theta=105^{\circ}$ were set equal to their values at $105^{\circ}$. Although this particular choice for the back hemisphere is not critical to the evaluation of gain from (2) and (5) because the power radiated into the back hemisphere is a small fraction of the total power radiated, some reasonable choice for the back-hemisphere pattern must be made to evaluate the gain reasonably accurately from (14) (or more precisely, from (14) with its integrand so modified in the back hemisphere).

The determination of gain using the approximate formulas (2) and (8) is immediately accomplished once the constant $C_{0}$ is computed from (11) as discussed in Sec. II-B-2. The expression for gain using (2) and (8) was given in (12b) and need not be repeated here. Gain curves computed from (12b) as well as (14) are drawn in figure 7 , which shows the considerable improvement in gain values of both (12b) and (14) over the gain determined from the classical Stratton-Chu resuits [2]. The comparison of measured and predicted values of gain in figure 7 indicates that the formulas (12b) and (14) predict gain with an accuracy of about $0.2 \mathrm{~dB}$.

\section{CONCLUSION}

This work was motivated by the need for simple, accurate expressions for the far fields and gain of open-ended rectangular waveguide probes used in making near-field antenna measurements. Except near the E-plane, previous expressions based on either the Stratton-Chu formulas or first-order geometrical edge diffraction predict patterns that deviate considerably from measured patterns--to the extent that their predicted gain values are 2 to $3 \mathrm{~dB}$ too low. The alternative expressions for the far fields developed here, reduce 
the previous limits of accuracy by about a factor of 10 , over the recommended usable bandwidth of $X$-band and larger standard rectangular waveguide. For Kuband and smaller standard waveguide, the wall thickness becomes an appreciable fraction of the waveguide cross-sectional dimensions and the approximate formulas may become less accurate.

Two methods were presented for deriving the alternative approximate formulas for the far fields (and the corresponding absolute gain). Both methods began by assuming the $\sin \phi-\cos \phi$ dependence of eq (1) with an E-plane pattern (2) given by the classical result of integration with the Stratton-Chu formulas. The two methods differed, however, in their determination of the $\mathrm{H}-$ plane pattern. The first method used an E-field aperture integration (3) of the $T E_{10}$ mode to derive the $H-p l a n e$ pattern (5) valid in the forward hemisphere only, whereas the second method relied upon modifying the physical optics result (6) of the Stratton-Chu formulas with a fringe current contribution to derive the H-plane pattern (8) valid for all far-field angles. A good estimate of the fringe current as an isotropic line source was finally obtained through a numerical solution to the electric field integral equation (EFIE) in Appendix I. (An alternative approach of estimating the fringe currents by the corresponding fringe currents of the perfectly conducting half plane gave excellent results in Appendix II for the open-ended parallel-plate waveguide, but failed to produce an $\mathrm{H}-\mathrm{plane}$ pattern to the desired accuracy for the rectangular waveguide.)

In brief, the two sets of approximate formulas emerged for the far fields--(2) and (5) or (2) and (8) inserted into (1). The former set, (2) and (5), approximates the far fields in the forward hemisphere without requiring the $\mathrm{TE}_{10}$ reflection coefficient for the open-ended waveguide, but predicts too narrow an $\mathrm{H}-\mathrm{pl}$ ane pattern for angles near $90^{\circ}$ off boresight and slightly less reliable gain values. The latter set, (2) and (8), requires the reflection coefficient, but yields a reliable estimate of the H-plane pattern for all angles of the far field including the back hemisphere. 


\section{APPENDIX I}

\section{THE ELECTRIC FIELD INTEGRAL EQUATION (EFIE) SOLUTION}

Consider the open-ended waveguide depicted in figure 1. Assume perfectly conducting walls $W$, infinitesimally thin, carrying a total surface current density $\bar{k}$. The electric field everywhere, except within the waveguide walls, can be expressed in terms of the vector and scalar potentials as

$$
-4 \pi i \omega \varepsilon_{0} \bar{E}(\bar{r})=k^{2} \int_{W} \bar{K}\left(\bar{r}^{\prime}\right) \psi\left(\bar{r}, \bar{r}^{\prime}\right) d S^{\prime}+\nabla \int_{W}\left(\nabla_{S}^{\prime} \cdot \bar{K}\left(\bar{r}^{\prime}\right)\right) \psi\left(\bar{r}, \bar{r}^{\prime}\right) d S^{\prime},
$$

where $\psi\left(\bar{r}, \bar{r}^{\prime}\right)$ is the scalar Green's function, $e^{i k\left|\bar{r}-\bar{r}^{\prime}\right|} /\left|\bar{r}-\bar{r}{ }^{\prime}\right|$.

Divide $\bar{K}$ into the sum of the physical optics current $\bar{K}_{10_{-}}$of the $T E_{10}$ mode (incident plus reflected) and the remaining "fringe current" $\bar{K}_{f}$ :

$$
\bar{k}=\bar{K}_{10}+\bar{K}_{f} \text {, }
$$

to recast $(A 1)$ into the form

$$
\begin{aligned}
-4 \pi i \omega \varepsilon_{0} \bar{E}(\bar{r}) & =k^{2} \int_{W} \bar{K}_{f}\left(\bar{r}^{\prime}\right) \psi\left(\bar{r}, \bar{r}^{\prime}\right) d S^{\prime}+\nabla \int_{W}\left(\nabla_{s}{ }^{\prime} \cdot \bar{K}_{f}\right) \psi d S^{\prime} \\
& +k^{2} \int_{W} \bar{K}_{10} \psi d S^{\prime}+\nabla \int_{W}\left(\nabla_{s}{ }^{\prime} \cdot \bar{K}_{10}\right) \psi d S^{\prime}
\end{aligned}
$$

The second line of (A3) represents the electric field produced by the truncated $\mathrm{TE}_{10}$ mode currents, i.e., the physical optics current. They can be conveniently expressed in terms of an integral of the fields (4) of the TE 10 mode in the infinite waveguide over only the aperture $A$ of the mouth of the open-ended waveguide, by means of the Stratton-Chu equations [1, Sec. 8.14]: 
$\begin{aligned} & (\bar{r} \text { inside } W+A) \\ & (\bar{r} \text { outside } W+A)\end{aligned}\left\{\begin{array}{c}-4 \pi i \omega \varepsilon_{0} \bar{E}_{10}(\bar{r}) \\ 0\end{array}\right\}=k^{2} \int_{W} \bar{K}_{10} \psi d S^{\prime}+\nabla \int_{W}\left(\nabla_{S}{ }^{\prime} \cdot \bar{K}_{10}\right) \psi d S^{\prime}$

$$
+\int_{A}\left[k^{2}\left(\hat{n} \times \bar{H}_{10}\right) \psi-i \omega \varepsilon_{0}^{\prime}\left(\hat{n} \times \bar{E}_{10}\right) \times \nabla^{\prime} \psi\right] d S^{\prime}
$$

(As an aside, note that for $\bar{r}$ outside the waveguide, the integral over $A$ in (A4) is the electric field calculated from the Stratton-Chu formulas applied to the truncated waveguide to obtain, in particular, the far fields (2) and (6). Thus, (A4) proves that the far fields obtained by Risser [2] from the Stratton-Chu formulas are those produced by the truncated $T_{10}$ mode currents. In other words, for $\bar{r}$ outside the waveguide, the $\bar{K}_{10}$ integrals in (A3) produce the same electric field as the Stratton-Chu formulas.) Substitute (A4) into (A3), let the observation point $\bar{r}$ approach the surface of the waveguide $W$ (from inside or outside $W$ ), take $\hat{n} \times$ the resulting equation (where $\hat{n}$ is the normal to $W+A$ into the waveguide) and set $\hat{n} \times \bar{E}$ and $\hat{n} \times \bar{E}_{10}$ equal to zero on $W$, to get

$$
\begin{aligned}
& 4 \pi i \omega \varepsilon_{0} \hat{n} \times \bar{E}_{i n c}(\bar{r})=\hat{n} \times[k^{2} \underbrace{}_{W} \bar{K}_{f} \psi d S^{\prime}+\nabla \underset{W}{g}\left(\nabla_{S}{ }^{\prime} \cdot \bar{K}_{f}\right) \psi d S^{\prime}], \\
& (\bar{r} \text { on } W)
\end{aligned}
$$

where $\bar{E}_{i n c}$ is defined as

$$
\bar{E}_{i n C}(\bar{r}) \equiv-\frac{1}{4 \pi i \omega \varepsilon_{0}} \int_{A}\left[k^{2} \hat{e}_{z} \times \bar{H}_{10} \psi-i \omega \varepsilon_{0}\left(\hat{e}_{z} \times \bar{E}_{10}\right) \times \nabla^{\prime} \psi\right] d S^{\prime},
$$

and $\oplus$ denotes the principal-value integral [16].

Equation (A5) is the desired EFIE applied to the open-ended rectangular waveguide. It has been derived here in a form that allows the aperture $\mathrm{TE}_{10}$ mode fields in (A6) to produce entirely the driving or incident electric field $\bar{E}_{i n c}$ on the left of (A5). Thus the unknown surface current densities $\bar{K}_{f}$ in (A5) are merely the remaining fringe currents that decay rapidly away from the 
end of the waveguide, provided, of course, the reflection coefficient is supplied with the $T E_{10}$ mode fields given explicitly in (4).

The incident field was calculated numerically from (A6) with measured reflection coefficient $\Gamma$ inserted into (4). The integral equation (A5) was then solved numerically for $\bar{K}_{f}$ on a scientific digital computer using a moment-method numerical scheme similar to that used by Glisson and Wilton [8]. Symmetry of the rectangular waveguide and $T E_{10}$ mode, which can be expressed easily by noting that a perfect electric and magnetic conducting plane can be inserted at $y=0$ and $x=0$, respectively, without changing the problem [9], allowed (A5) to be rewritten in terms of fringe currents over only one quarter of the waveguide. This reduced the number of unknowns by the same factor of four. The final resulting coefficient matrix of the unknown current $\bar{K}_{f}$ was inverted to find numerical values of $\bar{K}_{f}$. The numerical values of $\bar{K}_{f}$ were then inserted into the $\bar{K}_{f}$ integrals of (A3) for $r \rightarrow \infty$ in order to get the fringe-current contribution to the far fields to be added to the StrattonChu far fields [2], which equals, as proven above, the contribution of the $\bar{K}_{10}$ integrals in (A3) as $r \rightarrow \infty$.

To get far field patterns over the whole forward hemisphere to the desired few percent accuracy, this numerical EFIE solution required computer storage larger than the available central memory of the particular scientific computer at our disposal and a prohibitive amount of computer execution time. However, numerical experimentation with the computer solution revealed that the fringe currents contributing to the far-field $\mathrm{H}$-plane could be approximated surprisingly well by isotropic line sources in the y-direction at the edges $x= \pm 2 / 2$. It is this result that allowed the simplified fringecurrent solution in SeC. II-B-2 of the main text. 


\section{APPENDIX II}

\section{FAR FIELDS AND GAIN OF OPEN-ENDED PARALLEL-PLATE WAVEGUIDE}

The far fields and gain of the open-ended parallel-plate waveguide (figure 1 with $b \rightarrow \infty, \phi=0$ and $-\pi \leqslant \theta \leqslant \pi$ ) excited by the $T E_{10}$ mode can be found simply and accurately from the half-plane solution once the reflection coefficient is determined (e.g., from the exact solution [10,15] or from ray theory methods [11]). We begin with the far fields $\left(\bar{E}_{1}, \bar{H}_{1}\right)$ of the open-ended parallel-plate waveguide approximated by the aperture integration of the twodimensional Stratton-Chu formulas with the $T E_{10}$ mode fields (4):

$$
\begin{aligned}
& \bar{E}_{1}(r \rightarrow \infty, \theta)=F(r, \theta) \hat{e}_{y} \\
& \bar{H}_{1}(r \rightarrow \infty, \theta)=-\frac{F(r, \theta)}{Z_{0}} \hat{e}_{\theta},
\end{aligned}
$$

where

$$
\begin{aligned}
F(r, \theta) & =\frac{E_{0} k a \sqrt{2 \pi}}{8}\left[(1+\Gamma) \cos \theta+\frac{\beta}{k}(1-\Gamma)\right] \\
& \cdot\left[\frac{\cos \left(\frac{k a}{2} \sin \theta\right)}{\left(\frac{\pi}{2}\right)^{2}-\left(\frac{k a}{2} \sin \theta\right)^{2}}\right] \frac{e^{i\left(k r-\frac{\pi}{4}\right)}}{\sqrt{k r}} .
\end{aligned}
$$

Appendix I proved that the Stratton-Chu aperture integration of the $\mathrm{TE}_{10}$ mode fields gave the fields exterior to the waveguide and, in particular, the far fields produced by the truncated $T E_{10}$ mode currents, i.e., the far fields of the physical optics current of the incident plus reflected $\mathrm{TE}_{10}$ mode. Thus the fields in (A7) neglect only the contribution from the fringe currents (total current minus physical optics current, or equivalently, the evanescent mode current plus the exterior surface currents). For the parallel-plate 
waveguide, these fringe currents can be estimated quite accurately from the corresponding fringe currents of the half-plane problem.

Now the remaining electric far field of the conducting half-plane, after the far field of the physical optics current is subtracted, can be shown to be exactly $[12,13]$ :

$$
\bar{E}_{h p}(r \rightarrow \infty, \theta)=\frac{-\sin \frac{|\alpha|}{2} E i e^{i\left(k r-\frac{\pi}{4}\right)}}{\left[\cos \frac{\theta}{2}+\cos \frac{\alpha}{2}\right] \sqrt{2 \pi k r}} \hat{e}_{y} \quad\left(\begin{array}{c}
0 \leqslant|\alpha| \leqslant \pi \\
\alpha, \theta \neq \pm \pi
\end{array}\right),
$$

for a plane wave of amplitude $\hat{\mathrm{e}}_{\mathrm{y}}$ incident on the edge and propagating at an angle $\alpha$ from the conducting half-plane. For the parallel-plate waveguide, approximate the incident field on each half plane by the two plane waves impinging on each edge, one from the incident $T E_{10}$ mode and one from the reflected $T E_{10}$ mode. These two incident plane waves make an angle $\alpha$ with the half plane of

$$
\alpha_{0}=\cos ^{-1} \frac{\beta}{k}=\cos ^{-1}\left(\sqrt{1-\left(\frac{\pi}{k a}\right)^{2}}\right)
$$

and

$$
\pi-\alpha_{0},
$$

respectively. Substituting the angles (A9) into (A8) for the two impinging plane waves, and adding the contribution from the two half-planes forming the open-ended parallel-plate waveguide, gives the following approximate expression for the electric far field excited by the fringe currents:

$$
\bar{E}_{f}(r \rightarrow \infty)=E_{0}\left[\frac{\sin _{0} \frac{\alpha_{0}}{2}}{\cos \frac{\theta}{2}+\cos \frac{\alpha_{0}}{2}}+\Gamma \frac{\cos \frac{\alpha_{0}}{2}}{\cos \frac{\theta}{2}+\sin \frac{\alpha_{0}}{2}}\right] \frac{\cos \left(\frac{k a}{2} \sin \theta\right)}{\sqrt{2 \pi k r}} e^{i\left(k r-\frac{\pi}{4}\right)} \hat{e}_{y} .
$$


The sum of (A10) and (A7a) then gives an approximate expression for the total far field $\bar{E}_{p p}$ of the open-ended parallel-plate waveguide (with the $T E_{10}$ mode E-field parallel to the waveguide walls):

$$
\bar{E}_{p p}(r \rightarrow \infty, \theta)=\bar{E}_{1}(r \rightarrow \infty, \theta)+\bar{E}_{f}(r \rightarrow \infty, \theta) \text {. }
$$

The on-axis gain $G_{p p}$ defined with respect to an infintely extended line source is

$$
G_{p p} \equiv \frac{\pi r\left|\bar{E}_{p p}(r \rightarrow \infty, 0)\right|^{2}}{Z_{0}{ }_{p p}}=\frac{4 \pi r\left|E_{p p}\right|^{2}}{a\left|E_{0}\right|^{2} \frac{\beta}{k}\left(1-|\Gamma|^{2}\right)},
$$

since the input power $P_{p p}$ per unit length of the $T E_{10}$ mode is

$$
P_{p p}=\frac{a\left|E_{0}\right|^{2} \frac{\beta}{k}\left(1-|\Gamma|^{2}\right)}{4 Z_{0}} .
$$

Approximate far field patterns derived from (A11) (with (A7a) and (A10) inserted), and approximate gain values from (A12), are plotted in figures 8 and 9. The comparisons contained in figures 8 and 9 with the exact solution $[10,14,15]$ show the significant improvement of the approximate solution (A11) (which estimates the fringe current contribution as explained above) over that of the Stratton-Chu aperture integration (A7) (which neglects the fringe currents). 


\section{ACKNONLEDGMENT}

Allen C. Newe11, David A. Hill, and Ronald C. Wittmann of the National Bureau of Standards (NBS), and Professor Jung-Woong Ra of the Korea Advanced Institute of Science and Technology contributed generously to this work through their helpful discussions and reviews. Allen C. Newell, Douglas P. Kremer, Stanley B. Kilgore, and Bill C. Yates of NBS performed and supplied the far-field, gain, and reflection coefficient measurements of the open-ended rectangular waveguide probes. 


\section{REFEREMCES}

[1] Stratton, J. A. Electromagnetic Theory. New York, NY: McGraw-Hill; 1941. 615 p.

[2] Risser, J. R. Waveguide and Horn Feeds, chapter 10 in Microwave Antenna Theory and Design. Samuel Silver, ed. New York, NY: McGrawHi 11; 1949. 234-387.

[3] Ryan, C. E., Jr.; Rudduck, R. C. Radiation patterns of rectangular waveguides. IEEE Trans. Antennas and Propag., Vol. AP-16: 488-489; 1968 July.

[4] Johnson, C. C. Field and wave electrodynamics. New York, NY: McGraw$\mathrm{Hi} 1 \mathrm{1} ; 1965$. Eq. 10.26 .

[5] a) Yaghjian, A. D. Upper-bound errors in far-field antenna parameters determined from planar near-field measurements, Part I: Analysis. Nat. Bur. Stand. (U.S.) Tech. Note 667; 1975 October. Sec. II.A.3.

b) Yaghjian, A. D. Efficient computation of antenna coupling and fields within the near-field region. IEEE Trans. Antennas and Propag., Vol. AP-30: 113-128; 1982 January.

c) Yaghjian, A. D. Near-field antenna measurements on a cylindrical surface: A source scattering-matrix formulation. Nat. Bur. Stand. (U.S.) Tech. Note 696; 1977, September. Sec. 4.2.1.

[6] Kerns, D. M. General formula for voltage induced in a receiving antenna. Digest of the national radio science meeting; 1978 November; Boulder, C0. p. 14.

[7] Hess, D. W. Practica? considerations for near-field measurements and the use of probe correction. Digest of national radio science meeting; 1982 January; Boulder, C0. p. 186.

[8] Glisson, A. W.; Wilton, D. R. Simple and efficient numerical methods for problems of electromagnetic radiation and scattering from surfaces. IEEE Trans. Antennas and Propag., Vol. AP-28: 593-603; 1980 September.

[9] Tsai, L. L.; Dudley, D. G.; Wilton, D. R. Electromagnetic scattering by a three-dimensional conducting rectangular box. J. Appl. Phys., Vol. 45: 4393-4400; 1974 October.

[10] Weinstein, L. A. The theory of diffraction and the factorization method. Boulder, C0: Golem; 1969. Ch. 1.

[11] Yee, H. Y.; Felsen, L. B.; Keller, J. B. Ray theory of reflection from the open end of a waveguide. Siam J. Appl. Math., Vol. 16: 268-300; 1968 March. 
[12] Ufimtsev, P. Ia. Approximate computation of the diffraction of plane electromagnetic waves by certain bodies, Part I and II. Sov. Phys.Tech. Phys: 1708-1718, 1957; 2386-2396, 1958.

[13] Yaghjian, A. D.; Ra, J. W. Fringe-current diffraction coefficients for the half plane. Unpublished Nat. Bur. Stand. (U.S.) notes; 1982 September.

[14] Heins, A. E. The radiation and transmission properties of a pair of semi-infinite parallel plates--I. Quart. Appl. Math., Vol VI: 157$166 ; 1948$ JuTy.

[15] Marcuvitz, N., ed. Waveguide handbook. New York: McGraw-Hi17; 1951. Ch. 4.

[16] Yaghjian, A. D. Augmented electric and magnetic-field integral equations. Radio Science, Vo1. 16: 987-1001; 1981, November - December. 


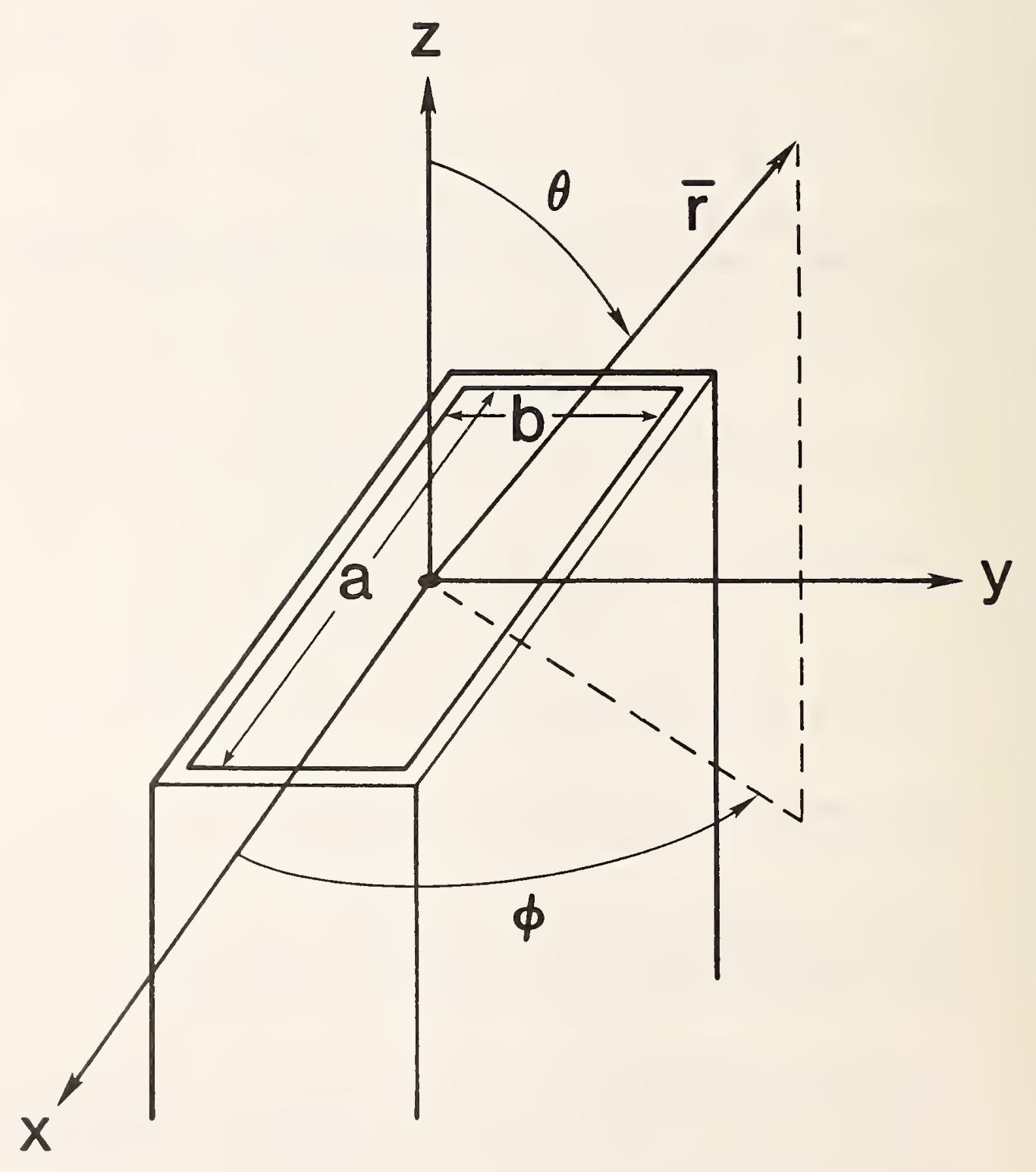

Fig. 1. Geometry of open-ended rectangular waveguide. 


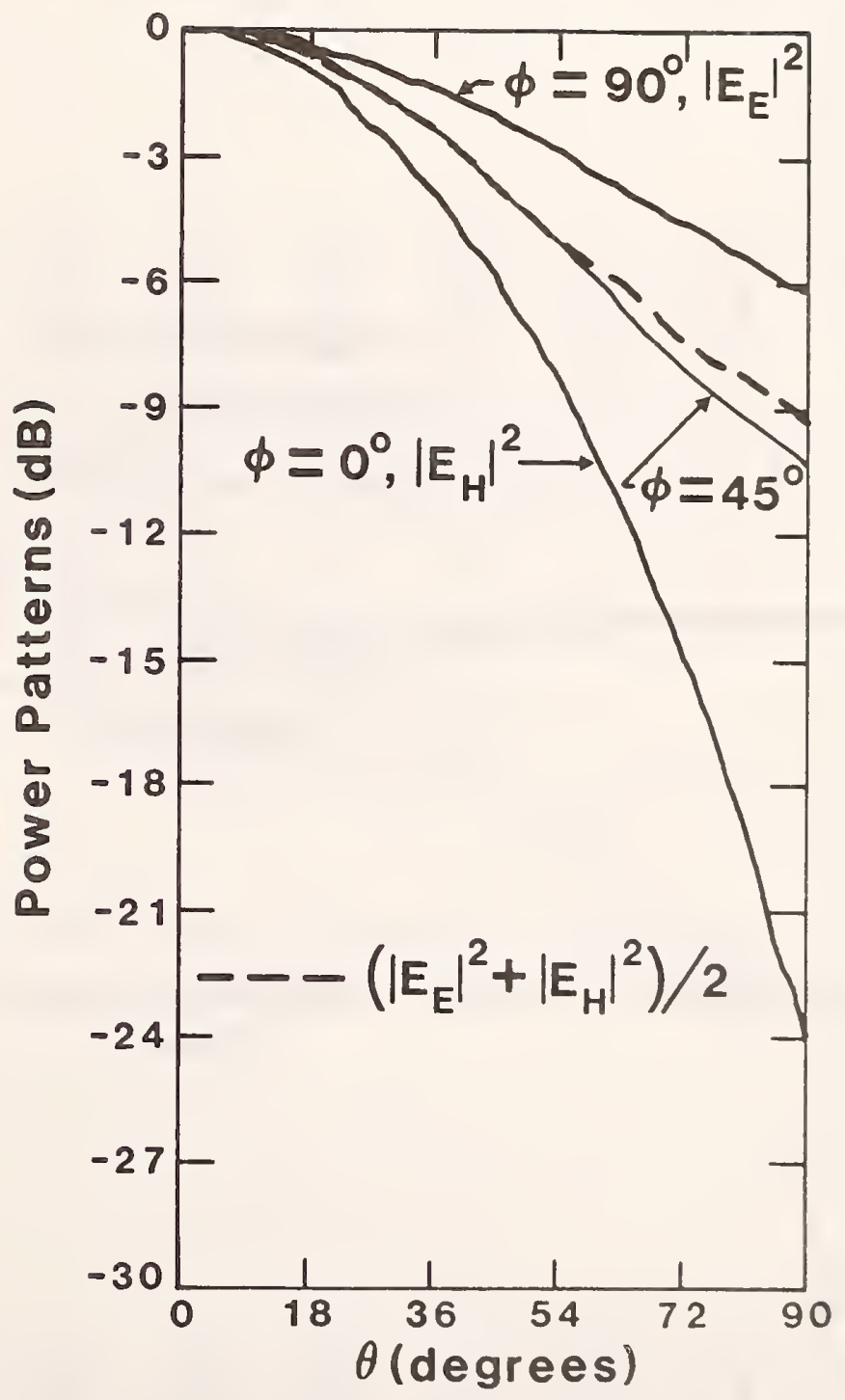

Fig. 2. Measured $E_{E}$ and $E_{H}$ power patterns of open-ended rectangular $X$-band waveguide at $9.32 \mathrm{GHz}$, and comparison of measured $\phi=45^{\circ}$ power pattern with $1 / 2\left(\left|E_{E}\right|^{2}+\left|E_{H}\right|^{2}\right)$ predicted by eq (1). 


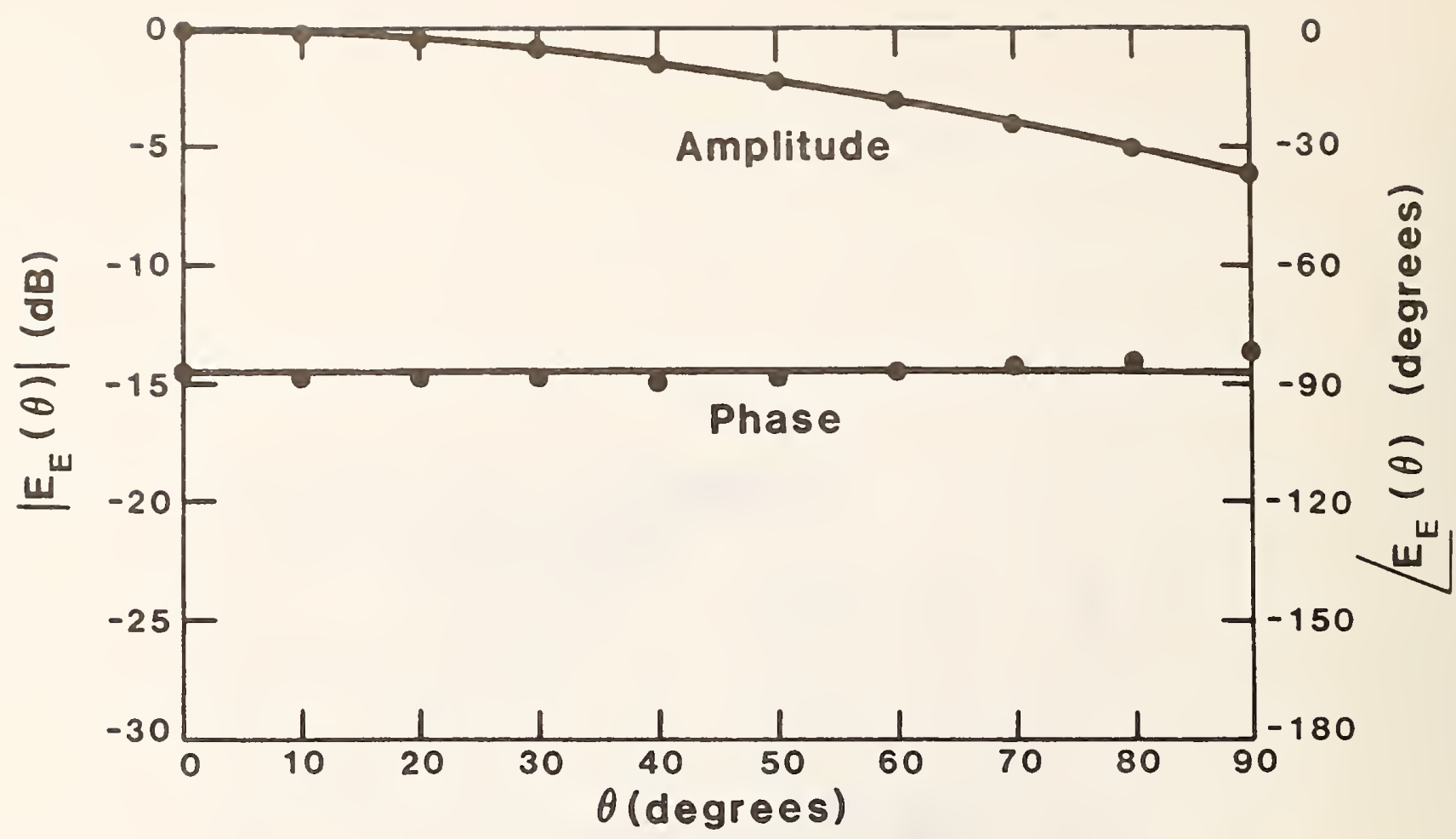

a) $X$-band rectangular waveguide at $9.32 \mathrm{GHz}$

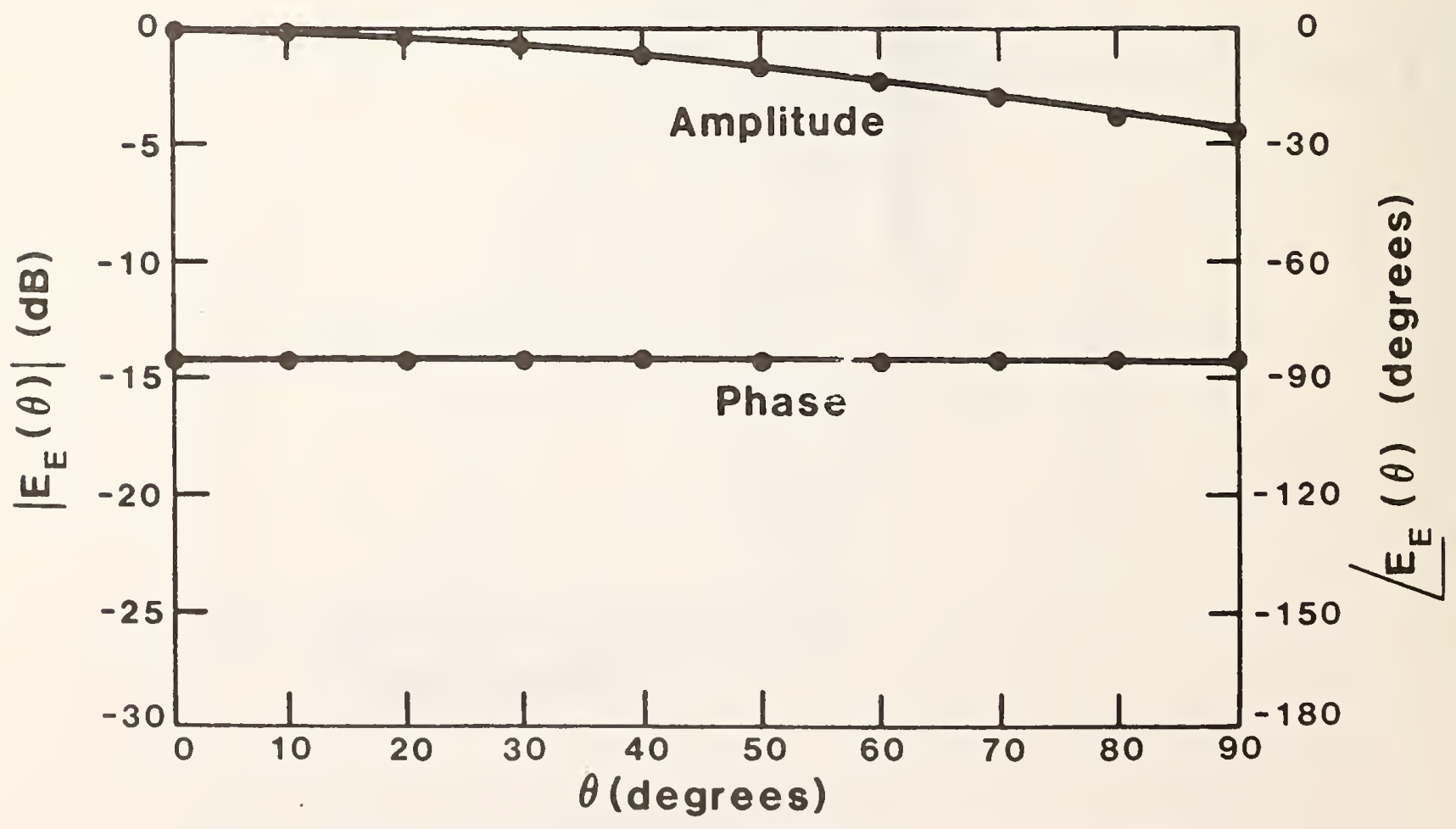

b) L-band rectangular waveguide at $1.0 \mathrm{GHz}$

Fig. 3. Amplitude and phase of E-plane field calculated from eq ), and measured at NBS (.....). 


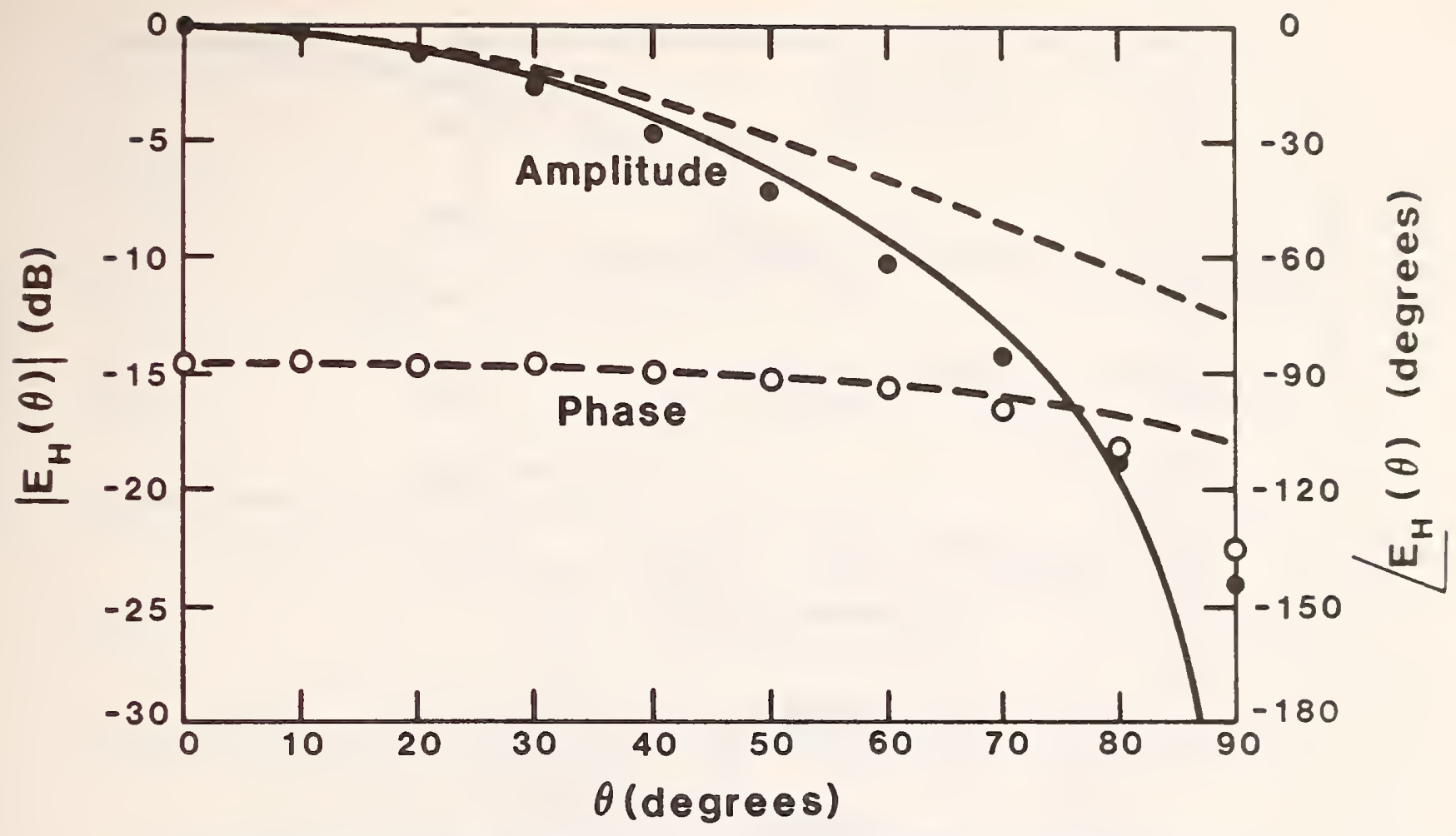

a) $\mathrm{X}$ - band rectangular waveguide at $9.32 \mathrm{GHz}$

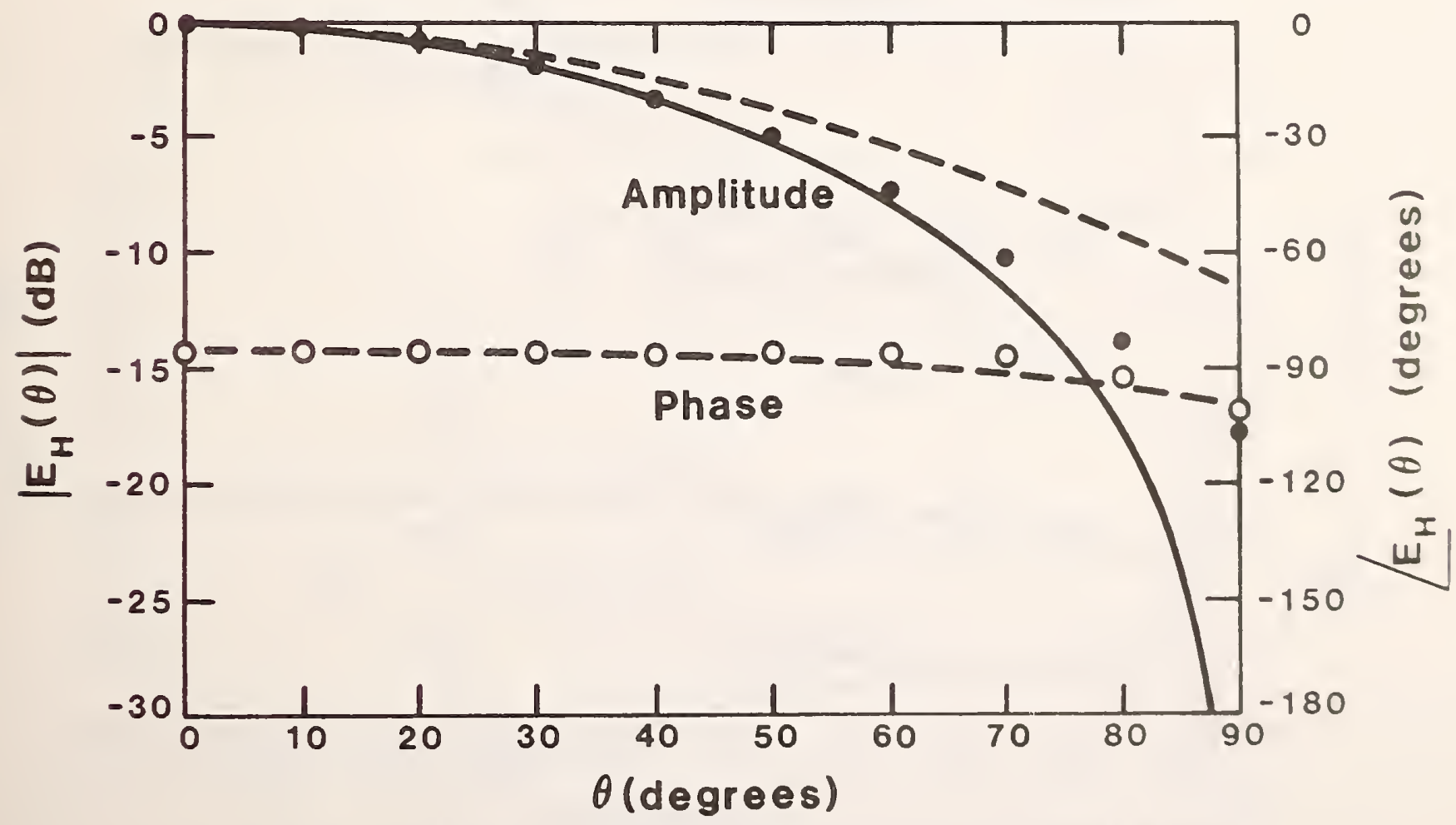

b) $\mathrm{L}$ - band rectangular waveguide at $1.0 \mathrm{GHz}$

Fig. 4. Amplitude and phase of H-plane field calculated from eq (5) ( and measured at NBS (.....). The H-plane phase from eq (5) is not shown because it is simply a constant phase near $-90^{\circ}$ found from eq (9). 

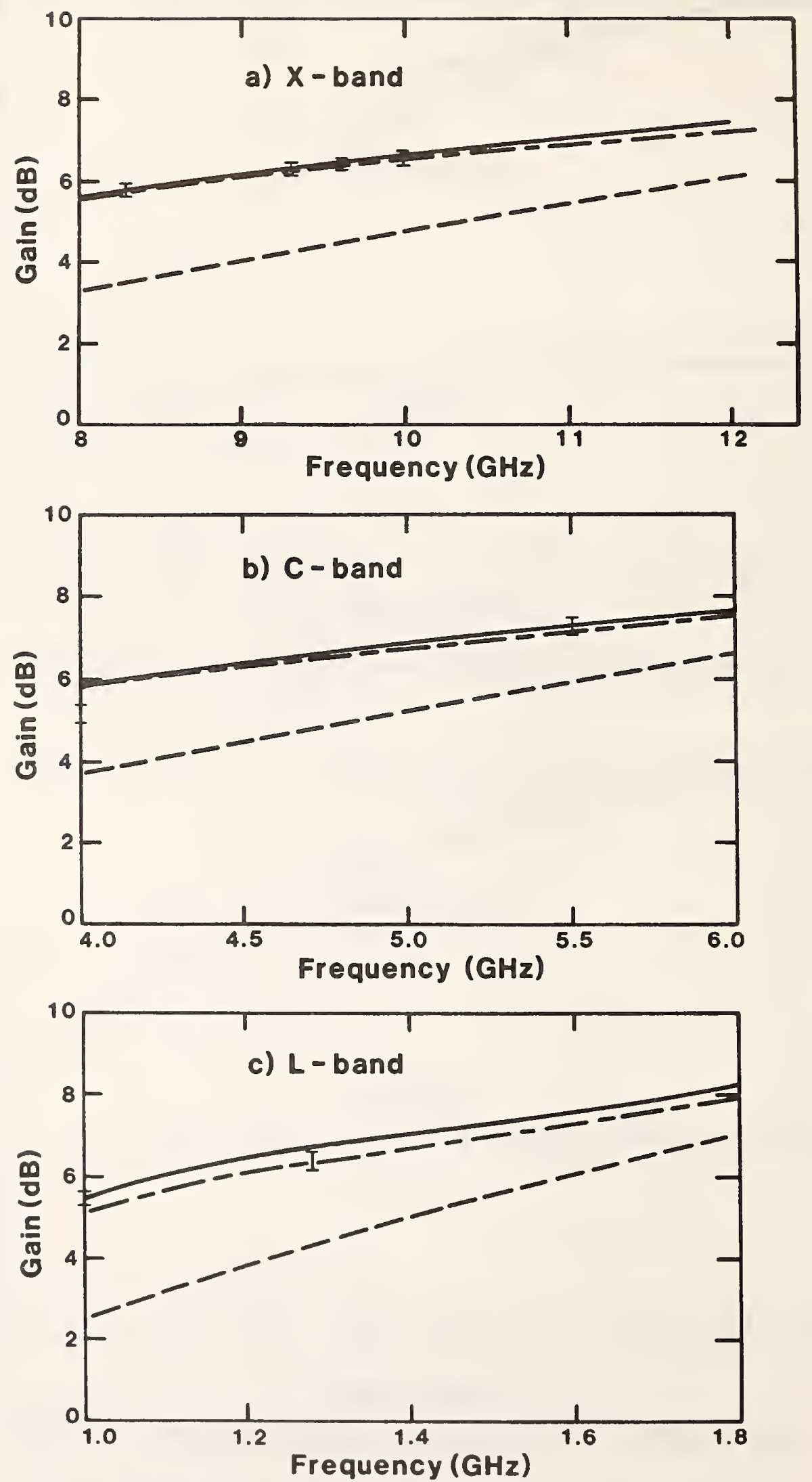

Fig. 7. On-axis gain of open-ended rectangular waveguide calculated from eq (12b) ( $)$, eq (14) ( _ _ - ), and the Stratton-Chu formulas (- - - - ). The I symbols denote NBS measured values of gain and estimated limits of error. 

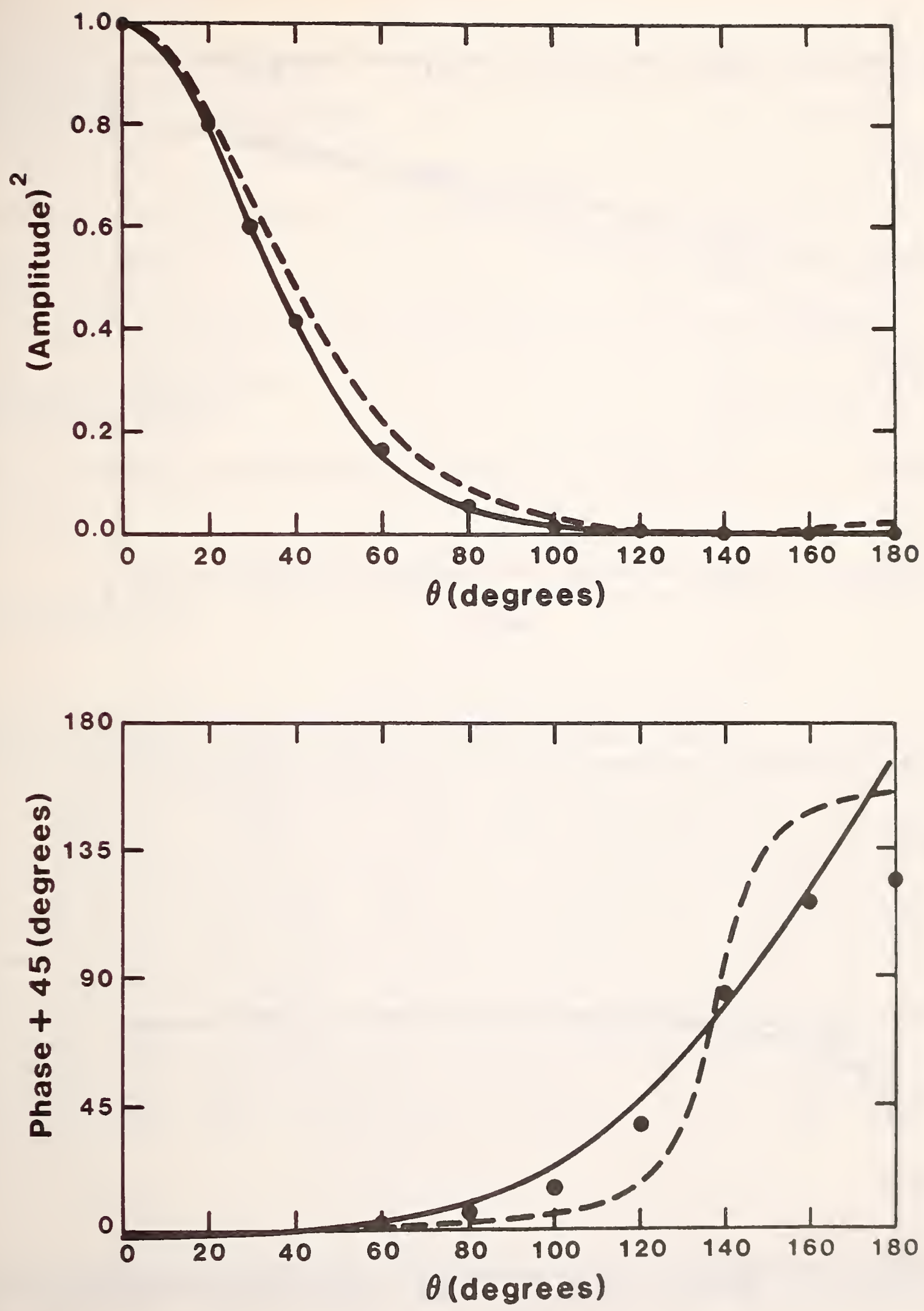

Fig. 8. Amplitude and phase of far field of open-ended parallel-plate waveguide $(a / \lambda=0.75)$ calculated from the exact solution

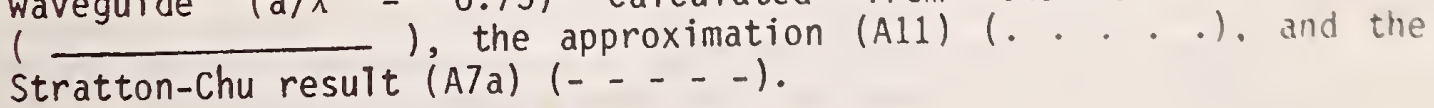



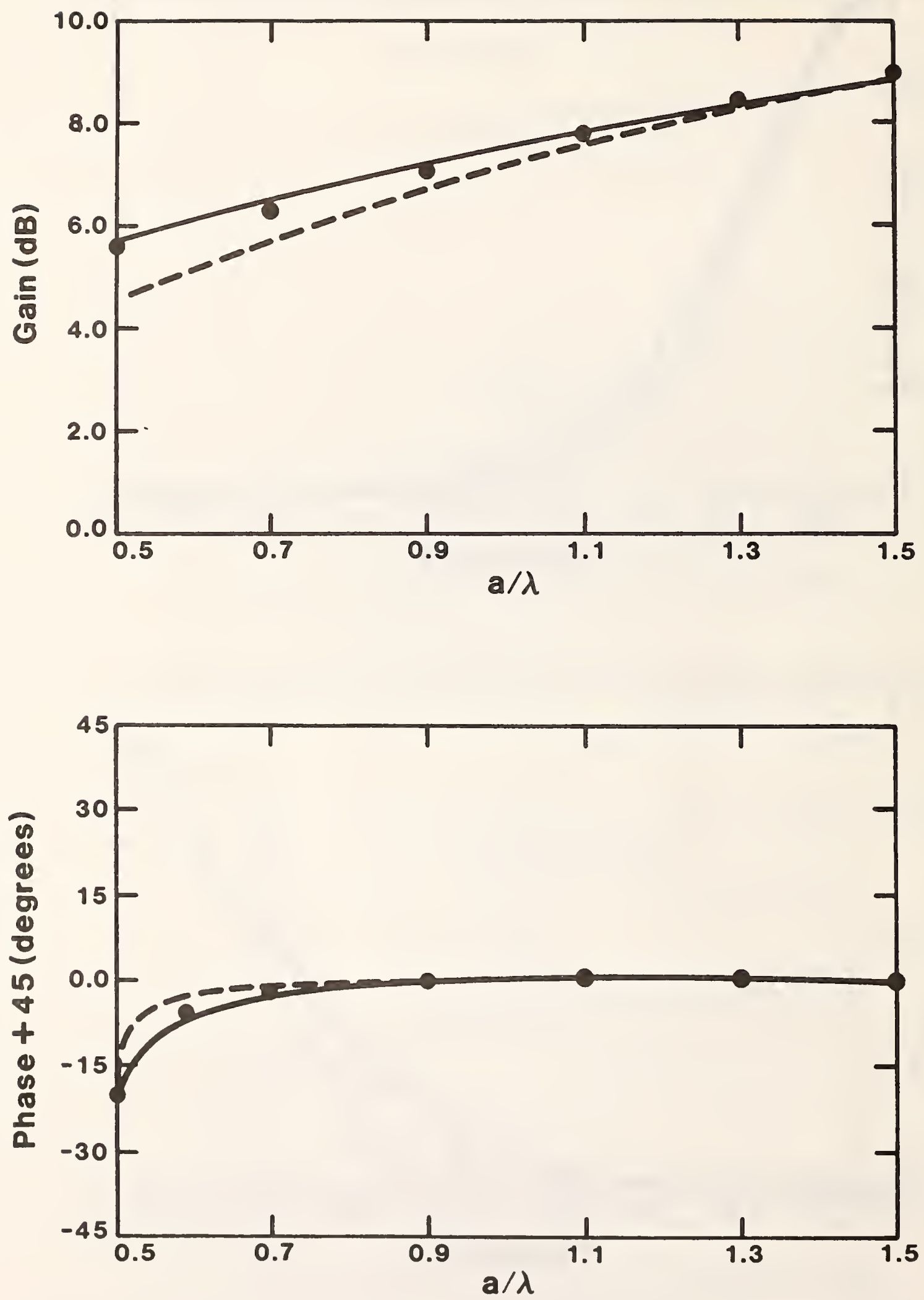

Fig. 9 On-axis gain and phase of open-ended parallel-plate waveguide calculated from the exact solution ( (A11) (....), and the Stratton-Chu result (A7a) ( - - -). 
NBS.114A (REV. 2.8C)

U.S. DEPT. OF COMM.

1. PUBLICATION OR REPORT NO.

BIBLIOGRAPHIC DATA

SHEET (See instructions)

NBSIR 83-1689

4. TITLE AND SUBTITLE

Approximate Formulas for the Far Fields and Gain of Open-Ended Rectangular Waveguide

\section{Arthur B. Yaghjian}

6. PERFORMING ORGANIZATION (If joint or other than NBS, see instructions)

7. ContracUGrant No.

NATIONAL BUREAU OF STANDARDS

DEPARTMENT OF COMMERCE

WASHINGTON, D.C. 20234

8. Type of Report \& Period Covered

9. SPONSORING ORGANIZATION NAME AND COMPLETE ADDRESS (Street, City, Stote, ZIP)

Same as \#6.

10. SUPPLEMENTARY NOTES

Document describes a computer program; SF-185, FIPS Software Summary, is attached.

11. ABSTRACT (A 200-word or less foctuol summory of most significont informotion. If document includes o significont bibliogrophy or literoture survey. mention it here)

Approximate formulas are derived for the far field and gain of standard, openended, unflanged, rectangular waveguide probes operating within their recommended usable bandwidth of frequencies. (Such probes are commonly used in making probecorrected near-field antenna measurements.) The formulas, which yield forward farfield power patterns and on-axis gains of $X$-band and larger waveguide probes to within about $2 \mathrm{~dB}$ and $0.2 \mathrm{~dB}$ accuracy, respectively, assume $(\sin \phi-\cos \phi)$ azimuthal angular dependence and an E-plane pattern given by the traditional aperture integration of the $\mathrm{TE}_{10}$ mode E- and H-fields in the Stratton-Chu equations. The H-plane pattern is estimated by two different methods. The first, and less accurate, method uses a purely Efield aperture integration to estimate the H-plane pattern. The second, more accurate, method uses an electric field integral equation (EFIE) to show that fringe currents near the shorter edges of the guide can be well-approximated by isotropic line sources. The amplitude of these line sources is then determined more simply by equating the total power radiated into free space to the net input power to the waveguide.

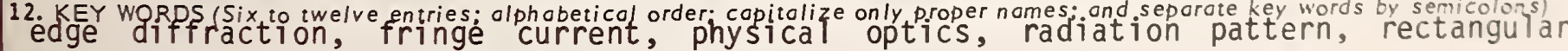
waveguide.

\section{AVAILABILITY}

XX Unlimited

For Official Distribution. Do Not Release to NTIS

Order From Superintendent of Documents, U.S. Government Printing Office, Washington, D.C. 20402.

X Order From National Technical Information Service (NTIS), Springfield, VA. 22I6I

14. NO. OF

PRINTED PAGES

40

15. Price

$\$ 8.50$ 


